\section{Repensando la selección de la carrera de Medicina desde los factores que inciden en la formación}

\author{
NADIA MUÑOZ N.. ,a, RENÉ BARRAZA L. ${ }^{2, b}$, \\ CRISTHIAN PÉREZ V. ${ }^{3, \mathrm{~b}}$, LILIANA ORTIZ M. ${ }^{3, \mathrm{c}}$
}

\section{Rethinking the selection of medical students, considering non-cognitive skills}

In Chile the only requirement to study medicine is to obtain an academic achievement score over a certain cutoff value. However, the literature states that this type of selection is insufficient, since the medical profession requires cognitive and non-cognitive skills. These abilities are associated with better adaptation and academic success, as well as less dropping out. Therefore, those skills should be considered in the selection process to assure that the education goals are met. The aim of this study was to review the existing literature regarding the selection and evaluation criteria for students who are applying to medical schools. It was evident that Chilean medical schools need to establish appropriate criteria to ensure a more inclusive and fair admission. They need to design a system of admission with solid evidence of validity and reliability, complementary to the current form of student selection. This system should be considered common to all schools of medicine and, in turn, consider the sensibilities of the particular mission of each school, since academic, cognitive, inter-and intrapersonal aspects may vary among them.

(Rev Med Chile 2015; 143: 1337-1342)

Key words: School Admission Criteria; Student Selection, Medical Schools.
'Oficina de Educación Médica,

Facultad de Medicina,

Universidad Católica del Norte,

Coquimbo, Chile.

Escuela de Psicología, Facultad de Ciencias Sociales, Universidad Santo Tomás, La Serena, Chile. ${ }^{3}$ Departamento de Educación Médica, Universidad de Concepción, Concepción, Chile. aMagíster en Educación Médica para Ciencias de la Salud.

bPsicólogo, Magíster en Psicología Educacional.

'Magíster en Educación Superior, M/Pedagogía Universitaria.

Trabajo financiado por Proyecto FONDECYT Regular 1121002.

Recibido el 31 de diciembre de 2014, aceptado el 29 de julio de 2015.

Correspondencia Dra Nadia Muñoz Navarro namunoz@ucn.cl

\section{Acerca del proceso de selección de postulantes} en educación superior

\section{I}

a masificación de la educación superior ha implicado el ingreso de alumnos con diversos niveles de desarrollo de sus habilidades de entrada, reflejando la pluralidad cultural y socioeducativa de sus respectivos orígenes ${ }^{1}$. La instalación de un sistema de selección de estudiantes por más de siete décadas, se ha sustentado en la premisa que no todos los egresados de la enseñanza secundaria cuentan con las habilidades, condiciones, capacidades, aptitudes o competencias para concluir estudios universitarios ${ }^{2}$. Así, el propósito del proceso de selección es certificar un nivel mínimo de desempeño, para aumentar la posibilidad de retención y éxito académico de los estudiantes ${ }^{3}$. No obstante, existe evidencia que indica que este sistema no sería del todo equitativo, marginando a los postulantes de sectores socioeconómicos y socioculturales menos favorecidos ${ }^{4}$. Esta realidad impone a las universidades, el desafío de perfeccionar sus prácticas para optimizar el proceso de formación, adaptación y selección de estos estudiantes, a fin de garantizar una educación con calidad y equidad ${ }^{5}$, por lo que, en los últimos años se han probado variadas fórmulas que permitan asegurar un acceso a la educación superior más igualitaria ${ }^{6}$.

Respecto a los procesos de selección de estudiantes para la educación superior, existe evidencia que señala como condiciones predictoras de per- 
manencia y logro académico: el origen socioeconómico, tipo de establecimiento de procedencia ${ }^{3,4}$ y el rendimiento académico previo ${ }^{7}$, no obstante respecto al rendimiento obtenido en pruebas estandarizadas, como la Prueba de Selección Universitaria (PSU), la literatura refleja posturas discrepantes respecto de su eficacia como mecanismo de selección ${ }^{8,9}$, siendo hasta la incorporación del ranking de notas de enseñanza media, casi el único dispositivo usado por las universidades en Chile para acceder a la educación superior.

\section{La selección en la carrera de Medicina}

En el caso de Medicina, la literatura reconoce que debe seguirse un exigente proceso de selección y acompañamiento a los postulantes, estableciendo ciertas condiciones de entrada que operan en diverso grado como predictores de rendimiento y logro académico en Medicina, entre ellas se encuentran: el rendimiento escolar previo ${ }^{10,13}$ y conocimientos académicos específicos, ${ }^{11}$ en materias asociadas a asignaturas biomédicas ${ }^{14}$ química, biología ${ }^{6}$ y otras más específicas como habilidades cognitivas ${ }^{6,14,15}$, inter e intrapersonales ${ }^{15,16}$ asociadas a características personales como integridad, responsabilidad y madurez ${ }^{16,17}$. Si bien este último grupo de condiciones no cuenta con evidencia concluyente ${ }^{12,13}$, su valor no radica sólo en el potencial predictivo de niveles de logro, sino en su uso como criterios prospectivos por su vinculación directa con aspectos del razonamiento clínico eficaz ${ }^{11,18}$.

En este sentido, se tiene claridad que el proceso de formación académica de un médico es uno de los más complejos y exigentes de la formación universitaria, y posiblemente no todos los aspirantes a Medicina presenten el conjunto de estas cualidades ${ }^{16}$. La mayoría de los programas de formación en Medicina enfrenta a los estudiantes de forma permanente a desafíos que, dependiendo de sus conductas de entrada, pueden estimular el desarrollo o constituirse en una dificultad para la adaptación y, en el peor de los casos, pudieran llevarle a la deserción ${ }^{19,20}$. Habitualmente se trata de personas que estaban altamente motivadas para estudiar la carrera, que en ocasiones hicieron más de un intento por ingresar y que descubren al poco tiempo que tienen pocas aptitudes para continuar los estudios y por ello su rendimiento es muy bajo ${ }^{21,22}$.
Si bien hoy se reconoce cuáles son los predictores del buen desempeño académico y permanencia en educación superior, específicamente en Medicina, los procesos de selección en Chile tienden a privilegiar mecanismos estandarizados tal como el puntaje PSU, pese a que no cuenta con evidencia concluyente respecto de su potencial como predictor de éxito, ni de aseguramiento de la permanencia del estudiante en el sistema o mecanismo de selección de habilidades propias de la profesión ${ }^{10}$.

Lo anterior marca la necesidad de contar con propuestas claras sobre qué otros factores deberían ser considerados al momento de seleccionar a los estudiantes y cuáles de estos, según la literatura, podrían ser predictores más adecuados de su desempeño como estudiantes de pregrado y futuros médicos.

\section{Características de entrada deseables de reconocer en los postulantes e instrumentos propuestos para su pesquisa en el proceso de selección}

De acuerdo a literatura, es posible clasificar en tres grupos las características de entrada asociadas al desempeño exitoso en Medicina: aquellas de orden académico, las de orden cognitivo y, por último, las asociadas a factores no cognitivos, como características intrapersonales y habilidades interpersonales, las cuales se describen a continuación.

\section{Aspectos de orden académico}

Dentro de los factores académicos se describen una multiplicidad de aspectos vinculados tanto al desempeño exitoso como a la permanencia de los estudiantes en la carrera. En cuanto al desempeño, uno de los más relevantes sería el rendimiento académico previo $^{10,11,12,13}$, a su vez, un alto nivel de conocimientos en materias específicas asociadas a asignaturas biomédicas ${ }^{13,14}$ como biología, matemáticas, física y química ${ }^{6,23}$, esto último presenta evidencia que indica que el conjunto de estas variables explicaría hasta $54 \%$ de la varianza en la predicción del desempeño académico futuro ${ }^{24}$. Otros aspectos de orden académico vinculados a la permanencia de los estudiantes de Medicina en la carrera, lo constituirían: el nivel de esfuerzo desplegado para rendir ${ }^{25}$, además del grado de conocimiento previo e interiorización acerca 
del programa de formación ${ }^{26}$, hábitos de estudio apropiados para el buen desempeño ${ }^{27}$, la cultura de aprendizaje del estudiante, las habilidades de trabajo en equipo y la apropiación y desarrollo del conocimiento $^{28,29}$.

En cuanto a la evaluación de estos aspectos, la literatura reporta el uso generalizado de instrumentos tales como examen de conocimientos basados en el nivel de conocimiento asociado a materias y contenidos vistos en la escuela secundaria, tales como: biología, química, física y matemáticas ${ }^{6,13,14,23}$, además de indicadores como promedio de notas asociados al rendimiento académico previo ${ }^{10,13}$. Finalmente, en cuanto a la pesquisa de aspectos académicos asociados a la permanencia, se reporta el uso de entrevistas orientadas a detectar aspectos vocacionales ${ }^{26}$ y escalas de autorreporte para la detección de hábitos y cultura académica del postulante ${ }^{20}$.

\section{Aspectos de orden cognitivo}

Los aspectos de orden cognitivo reseñados en la literatura hacen alusión a habilidades académicas y cognitivas específicas, tales como las habilidades de razonamiento verbal ${ }^{13,30}$, comunicación escrita ${ }^{24}$, capacidad de analizar racionalmente una situación problema $^{31}$, comprensión de textos científicos ${ }^{23}$, pruebas de habilidad mental general ${ }^{32}$.

En algunos países de la Unión Europea ${ }^{13,22,31,33}$, como de Oriente Medio ${ }^{11,24}$, se utilizan pruebas de habilidad cognitiva consistentes en ítems de desempeño en ámbitos verbales, numéricos o de figuras, para los cuales el postulante cuenta con un tiempo limitado para responder, además de otro tipo de evaluaciones como la redacción de cartas y escritos que permitan reconocer capacidad de expresión escrita del postulante en aspectos práxicos, como la grafía y formales del lenguaje, como ortografía y uso de la estructura gramatical.

La evaluación de los aspectos cognitivos ha demostrado tener relación directa con el aumento significativo de la probabilidad de progreso exitoso en la carrera y la disminución significativa de la proporción de abandono temprano ${ }^{22}$, además de presentar vinculación directa con aspectos cognitivos específicos del razonamiento clínico eficaz ${ }^{11}$ como la capacidad para identificar y recolectar información relevante, ya sea del contexto o de la tarea, capacidad para adaptar decisiones en tanto cambian las condiciones del problema, capacidad para procesar e interpretar una multiplicidad de datos y tomar decisiones con justificación ética ${ }^{18}$. Cada uno de estos elementos forma parte de las metas del proceso formativo que se busca desarrollar y para el cual el proceso de selección debe dar cuenta de forma certera.

\section{Aspectos de orden no cognitivo}

Este tipo de aspectos hacen referencia a las denominadas "habilidades blandas" ${ }^{13}$, dentro de las cuales se encuentran las características intrapersonales y las habilidades interpersonales. Entre los factores intrapersonales es posible encontrar dos grupos: el primero de ellos referido a aspectos vocacionales y motivacionales, los cuales al verse debilitados o pobremente definidos, impactan negativamente en el desempeño y progresión académica de los estudiantes ${ }^{19,20,21,26}$. En contrapartida, presentarán un mejor desempeño aquellos estudiantes que presenten altos niveles de engagement o compromiso académico, es decir a quienes sus estudios les generen mayor vigor, absorción, dedicación ${ }^{34}$ y una mayor motivación, toda vez que ésta determina el esfuerzo que se espera invertir en una tarea $^{35}$. Por otra parte, los aspectos referidos a la salud mental del estudiante, más específicamente la presencia de sintomatología ansiosa, depresiva o las estrategias de manejo del estrés ${ }^{36}$, afectarán no sólo la salud física, sino también pueden conducir a problemas académicos, reprobaciones e incluso a la deserción ${ }^{21,23,37}$.

Finalmente, respecto a las habilidades interpersonales, se menciona la inteligencia emocional ${ }^{12}$, los rasgos de personalidad ${ }^{12,17}$ y las habilidades de comunicación interpersonal ${ }^{13}$. Estos elementos guardan relación con la conciencia de las emociones y del impacto de éstas en el proceso de toma de decisiones, la capacidad para hacer frente a las emociones problemáticas y modular el estrés, además de la capacidad para mantener relaciones de trabajo efectiva y colaborativas ${ }^{18}$. Por otra parte, la evidencia da cuenta que la inclusión de este tipo de aspectos, además de los cognitivos y los antecedentes académicos, tienden a disminuir la deserción temprana, mejorando los estándares de rendimiento en diversos niveles ${ }^{13,38}$ y permanencia en la carrera ${ }^{15,37}$.

La evaluación de estos aspectos reporta la utilización de pruebas estandarizadas para la evaluación de la inteligencia emocional ${ }^{12}$ y la personalidad $^{12,17}$, además de otro tipo de evaluaciones 
como la exposición a situaciones interpersonales tras las cuales el evaluado debe enjuiciar la situación desplegando sus destrezas para discriminar adecuadamente la situación presentada ${ }^{13}$ y entrevistas de admisión ${ }^{13,39}$.

\section{Otros factores a considerar en el proceso de selección de postulantes}

Hasta aquí, la discusión de antecedentes presentados se ha centrado en las características del estudiante, sin embargo, la literatura evidencia la emergencia de otros aspectos que deben considerarse como: los antecedentes de los miembros del comité de admisión, puesto que son ellos quienes pueden influir en el proceso de selección, deliberación, votación y toma de decisión final de la admisión de un postulante ${ }^{12}$, lo cual podría eventualmente ejercer influencias indirectas en favor de ciertos grupos culturales, sociales o étnicos en favor de otros ${ }^{33,39}$. Por otra parte, se debe tener en cuenta la eficacia de las políticas de admisión, los procesos y criterios en los cuales descansa dicho proceso. En este sentido, se consigna que la misión propuesta por cada una de las escuelas de Medicina es un elemento central que debe guiar de forma integral el proceso y la revisión de antecedentes de los postulantes ${ }^{40}$. Finalmente, otros factores de interés incluyen la evaluación de aspectos vinculados al profesionalismo y factores de orden sociocultural, lo cuales no son habitualmente incluidos como parte de los procesos de admisión ${ }^{12,41}$.

\section{Discusión}

Tras la revisión de la literatura es posible constatar que, tanto en Europa, Oriente Medio y Latinoamérica, se presentan conclusiones orientadas a establecer que ciertas características de entrada de los postulantes a la carrera de Medicina, son más deseables que otras en un proceso de selección. Si bien pareciera apreciarse un patrón, en cuanto a que los antecedentes académicos tendrían mayor peso predictivo en el éxito académico ${ }^{10,11,12,13,14}$, estos resultados en su mayoría corresponden a investigaciones relativas a experiencias locales, donde los diseños utilizados no presentan un potencial explicativo definitivo, lo cual hace que la evidencia no sea concluyente, por lo que se re- quiere seguir investigando. Esto pone de relieve la necesidad de considerar las políticas de admisión de los distintos centros formadores de médicos, puesto que los procesos que se sigan deben probar su efectividad en distintas escuelas.

No obstante, un aspecto relevante de tener en cuenta es la consideración de la misión particular declarada por cada escuela de Medicina. En este sentido, la elección de un postulante, como el proceso mediante el cual se selecciona, debe considerar este ideario para proponer con claridad el tipo de estudiante con el cual trabajar. Estas características presentes en el postulante evaluado deberían ajustarse al ideario formativo que cada escuela de Medicina en particular profesa. Sin embargo, la preferencia por estudiantes con altos estándares académicos pareciera hacer olvidar esta cuestión central a la hora de seleccionar; en este sentido se propone considerar la elección de un postulante para un programa con un ideario formativo en específico, más que un postulante estándar que asegure logro académico en cualquier programa. Esto porque el éxito académico no es necesariamente sinónimo de éxito profesional, ni formación integral ${ }^{38}$.

En Chile, la selección de postulantes a Medicina ha sido tradicionalmente vía PSU, mecanismo cuestionado en cuanto a su potencial para establecer medidas predictivas fiables acerca del éxito académico del estudiante ${ }^{8,10}$, ya que se concentra en aspectos cognitivos declarativos, evaluando lo que el alumno sabe y no lo que puede hacer con ello ${ }^{10}$. Sólo en los últimos años se ha abierto la posibilidad de incorporar otros indicadores como el ranking de notas de enseñanza media ${ }^{9}$. No obstante la incorporación del ranking, el proceso de selección para la carrera carece de elementos evaluativos propios, no considerando aspectos sociales, intrapersonales, académicos y no académicos, lo cual impide ver a quiénes -ingresando al sistema- podrían necesitar más apoyo para tener éxito en su formación integral como médicos e igualmente podría estar dejando fuera, injustamente, a personas que podrían tener un mejor desempeño integral durante y después del pregrado ${ }^{10}$. En un contexto actual, con un estudiantado más heterogéneo, se vuelve esencial saber qué tipo de alumnado se enfrenta y cómo poder ayudarlos a terminar con éxito la carrera donde se les ha aceptado ${ }^{1}$.

Mientras el proceso de selección para Medicina 
no se modifique e incorpore los aspectos antes mencionados, surge la necesidad de realizar evaluaciones tardías a los postulantes seleccionados, a fin de reconocer y potenciar el nivel de habilidades de entrada que pudieran ser deseables para el logro del ideario formativo de las misiones particulares de cada escuela de Medicina. En esta misma línea, si uno de los criterios rectores para la admisión a la carrera fuese la misión particular de cada escuela, queda abierta la pregunta si se considerará aceptable asumir determinados riesgos, que se compensen con otras aptitudes, atributos, o rasgos que los postulantes posean y que sean deseables para el desarrollo del programa o la profesión.

Las escuelas de Medicina de Chile enfrentan el desafío de establecer vías apropiadas para garantizar criterios de admisión más inclusivos y justos, coherentes con los consensos internacionales relativos a la educación superior en general y la educación médica en particular. En el caso de nuestro país, si la selección de postulantes a la carrera sigue dependiendo de forma exclusiva de la PSU o de la incorporación del ranking, resulta un proceso de selección no sólo cuestionable, sino que insuficiente ${ }^{19}$. Esto demanda diseñar un sistema con evidencia sólida de su validez y confiabilidad para seleccionar estudiantes, que incorpore aspectos generales a todas a las escuelas de medicina, pero que también atienda a las sensibilidades de la misión particular de cada escuela y en cuyo caso sea posible brindar distintas ponderaciones a los aspectos académicos, cognitivos, inter e intrapersonales, los cuales forman parte de las metas del proceso formativo que se busca desarrollar y para el cual el proceso de selección debe dar cuenta de forma certera.

\section{Referencias}

1. Oficina para la Cooperación y el Desarrollo Económico. El Aseguramiento de la Calidad en la Educación Superior en Chile 2013. Paris: OCDE; 2013 Serie: Revisión de Políticas Nacionales de Educación No. 60496.

2. Donoso S, Schiefelbein E. Análisis de los modelos explicativos de retención de estudiantes en la universidad: una visión desde la desigualdad social. Estud. pedagóg. 2007; 33 (1): 7-27.

3. Román C. Más programas propedéuticos en Chile. El discurso de los estudiantes en la Universidad Católica Silva Henríquez. Estud. pedagóg 2013; 39 (2): 263-78.
4. Espinoza O, González L . Políticas de educación superior en Chile desde la perspectiva de la equidad. Revista Sociedad y Economía 2012; (22): 69-94.

5. Contreras M, Corbalán F, Redondo J. Cuando la suerte está echada: estudio cuantitativo de los factores asociados al rendimiento en la PSU. Revista Iberoamericana sobre Calidad, Eficacia y Cambio en Educación 2007; (5):259-63.

6. Rodríguez R, Díaz P, Moreno M, Bacallao J. Capacidad predictiva de varios indicadores de selección para el ingreso a la carrera de Medicina. Educ Med Super 2000; 14 (2): 128-35.

7. Contreras D, Gallegos S, Meneses F. Determinantes de Desempeño Universitario: ¿Importa la habilidad relativa? Revista Calidad de la Educación 2009; 30: 17-48.

8. Koljatic M, Silva M. Algunas reflexiones a siete años de la implementación de la psu. Revista Estudios Públicos 2010; 120: 125- 46.

9. Jil F, Paredes R, González I. El ranking de las notas: inclusión con excelencia. Santiago: Centro de políticas públicas UC; 2013. Serie temas de la agenda: 60. Disponible en: http://politicaspublicas.niceapp.cl/publicacion/ serie-temas-de-la-agenda/serie-no-60-el-ranking-de-lasnotas-inclusion-con-excelencia/

10. Pérez C, Ortiz L, Parra P. Prueba de Selección Universitaria, rendimiento en enseñanza media y variables cognitivo-actitudinales de alumnos de Medicina. Rev Educ Cienc Salud 2011; 8 (2): 120-7.

11. Murshid $\mathrm{K}$. The predictive value of individual admission criteria on academic performance in a Saudi medical college. Journal of Taibah University Medical Sciences 2013; 8 (1): 18-23.

12. Stratton T, Elam C. A holistic review of the medical school admission process: examining correlates of academic underperformance. Med Educ Online. [revista on line] 2014 Abril 1]; 19.

13. Lievens F. Adjusting medical school admission: assessing interpersonal skills using situational judgement tests. Med Educ 2013; 47 (2): 182-9.

14. Rodríguez M, Gómez V. Indicadores al ingreso en la carrera de medicina y su relación con el rendimiento académico. Revista de la educación superior 2010; 39 (153): 43-50.

15. Urlings-Strop LC, Stijnen T, Themmen AP, Splinter TA. Selection of medical students: a controlled experiment. Med Educ 2009; 43 (2): 175-83.

16. Az-zuhri M, Azwany N, Saiful M, Yusoff B, Noor S. Comparative Study on Medical Student Personality Traits between Interview and Non-interview Admission Method in University Sains Malaysia. Procedia-Social and Behavioral Sciences 2014; 116: 2281-5. 
17. Castaño J, Cerón A, Collazos AK, Molina A, Osorio J, Ospina A, et al. Factores que inciden en la motivación académica en un programa de Medicina, Manizales, Colombia, 2010. Archivos de Medicina (Col) 2012; 12 (1): 46-61.

18. Schwartz A, Elstein A Clinical reasoning in medicine. En: Higgs J, Jones M, Loftus S, Christensen N. editores, Clinical Reasoning in the Health Professions Philadelphia, USA; Elsevier Butterworth Heinemann: 2008. p 223-34.

19. López I, Marín G, García M. Deserción escolar en el primer año de la carrera de Medicina. Educ Med Super 2012; 26 (1): 45-52.

20. Hernández J, Hernández R, Nieto A, Hernández-Sierra J. Factores de riesgo para la deserción de estudiantes en la facultad de Medicina de la Universidad Autónoma de San Luis Potosí (UASLP), México. Gac Méd Méx 2005; 141 (5): 445-7.

21. Freites G, Hernández L, Delgado L, García B. Factores relacionados con la deserción escolar al programa nacional de formación del médico integral comunitario. Educ Med Super 2009; 23 (1): 1-13.

22. Reibnegger G, Caluba HC, Ithaler D, Manhal S, Neges HM, Smolle J. Progress of medical students after open admission or admission based on knowledge tests. Med Educ 2010; 44 (2): 205-14.

23. Labanino Y. Deserción escolar universitaria según el nuevo programa de formación de médicos en la comunidad Gambiana de Bansang. Medisan 2012; 16 (6): 870-80.

24. Al Alwan I, Al Kushi M, Tamim H, Magzoub M, Elzubeir M. Health sciences and medical college preadmission criteria and prediction of in-course academic performance: a longitudinal cohort study. Adv Health Sci Educ Theory Pract 2013; 18 (3): 427-38.

25. O'Neill LD, Wallstedt B, Eika B, Hartvigsen J. Factors associated with dropout in medical education: a literature review. Med Educ 2011; 45 (5): 440-54.

26. Rodríguez S, Hernández J, Duret M, Noa Y. Diagnóstico de orientación vocacional de estudiantes de Medicina del Policlínico Universitario de Nuevitas. Rev Hum Med 2011; 11 (1): 185-95.

27. Infante $\mathrm{N}$, Mendo N, Vázquez $\mathrm{M}$. Factores determinantes de la deserción escolar en el policlínico docente "frank país garcía”. MEDISAN 2012; 16 (4): 526-31.

28. Fasce E, Ortega J, Pérez C, Márquez C, Parra P, Ortiz L et al. Aprendizaje autodirigido en estudiantes de primer año de Medicina de la Universidad de Concepción y su relación con el perfil sociodemográfico y académico. Rev Med Chile 2013; 141 (9): 1117-25.

29. Yeh DD, Hwabejire JO, Imam A, Mullen JT, Smink
D, Velmahos G, et al. Survey of study habits of general surgery residents. J Surg Educ 2013; 70 (1): 15-23.

30. Makoul G, Curry RH. The value of assessing and addressing communication skills. JAMA 2007; 298 (9): 1057-9.

31. Simmenroth-Nayda A, Meskauskas E, Burckhardt G, Görlich Y. Medical school admission test at the University of Goettingen - which applicants will benefit? Z Evid Fortbild Qual Gesundhwes 2014; 108 (10): 609-17.

32. Kelly ME, Regan D, Dunne F, Henn P, Newell J, O’Flynn S. To what extent does the Health Professions Admission Test-Ireland predict performance in early undergraduate tests of communication and clinical skills? An observational cohort study. BMC Med Educ 2013; 13: 68.

33. O'Neill L, Vonsild MC, Wallstedt B, Dornan T. Admission criteria and diversity in medical school. Med Educ 2013; 47 (6): 557-61.

34. Parra P. Relación entre el nivel de Engagement y el rendimiento académico teórico/práctico. Rev Educ Cienc Salud 2010; 7 (1): 57-63.

35. Montalvo M, Armenta J, Gutiérrez F, Bazán I, Castañeda I, Chávez V. et al. Desempeño académico en el primer año de Medicina: relación con intereses vocacionales, actitud ante el estudio y fuerza de motivación. FEM 2013; 16 (2): 111-7.

36. Yusoff MS, Abdul Rahim AF, Baba AA, Ismail SB, Mat $\mathrm{Pa}$ MN, Esa AR. Prevalence and associated factors of stress, anxiety and depression among prospective medical students. Asian J Psychiatr 2013; 2: 128-33.

37. Yusoff MSB, Rahim AFA, Yaacob MJ. The prevalence of final year medical students with depressive symptoms and its contributing factors. International Medical Journal 2011; 4: 305-9.

38. Arora S, Russ S, Petrides K, Sirimanna P, Aggarwal R, Darzi A, et al. Emotional intelligence and stress in medical students performing surgical tasks. Acad Med 2011; 86 (10): 1311-7.

39. Puddey I, Mercer A, Carr S, Louden W. Potential influence of selection criteria on the demographic composition of students in an Australian medical school. BMC Med Educ 2011; 11: 97.

40. Simpson PL, Scicluna HA, Jones PD, Cole AM, O'Sullivan AJ, Harris PG, et al. Predictive validity of a new integrated selection process for medical school admission. BMC Med Educ 2014; 14: 86.

41. Addams AN, Bletzinger RB, Sondheimer HM, White SE, Johnson LM. Roadmap to diversity: integrating holistic review practices into medical school admission processes. Washington, DC: Association of American Medical Colleges; 2010. p 1-25. 